\title{
Mini Sling (Ophira) versus Pubovaginal Sling for Treatment of Stress Urinary Incontinence: A Medium-term Follow-up
}

\author{
Farzaneh Sharifiaghdas ${ }^{1}$, Mahmoodreza Nasiri', \\ Mahboubeh Mirzaei ${ }^{2}$, Behzad Narouie ${ }^{1,3}$ \\ ${ }^{1}$ Urology and Nephrology Research Center, Department of Urology, Shahid \\ Labbafinejad Medical Center, Shahid Beheshti University of Medical Sciences, \\ Tehran, Iran; \\ ${ }^{2}$ Department of Urology, Kerman University of Medical Sciences, Kerman, Iran; \\ ${ }^{3}$ Department of Urology, Zahedan University of Medical Sciences, Zahedan, Iran
}

Received November 7, 2014; Accepted September 15, 2015.

Key words: Mid-urethral mini sling - Autologous - Stress urinary incontinence Ophira

Abstract: To compare two different procedures, mid-urethral mini sling (Ophira) and autologous rectus fascia sling, according to their medium-term subjective and objective outcome and satisfaction rates in the treatment of stress urinary incontinence in women. One hundred women with the main complaint of stress urinary incontinence were randomized to be treated with either mini sling (Ophira) or autologous rectus facia pubovaginal sling. Preoperative evaluation consisted of: physical examination, blood biochemistry urine analysis and culture, urinary tract ultrasound scan, conventional multi-channel urodynamic study, cystourethroscopy, cough induced stress test and Incontinence Impact Questionnaire (IIQ). The patients were objectively and subjectively re-evaluated at 1, 3, 6 and 12 postoperative months and the last visit and the collected data of more than one year follow-up were compared with preoperative assessments. Seventy two out of one hundred patients were followed for a mean time of $13.8 \pm 4.4$ months (12-20 months range). Objective cure rate, according to cough-induced stress test was recorded in $88.6 \%$ and $89.2 \%$ of the mini sling (Ophira) and the rectus facia sling group respectively $(P=1.0)$. Postoperative mean IIQ score decreased to $42.7 \pm 11.4$ and $50.2 \pm 11.1$ in the mini sling (Ophira)

Mailing Address: Behzad Narouie, MD., Urology and Nephrology Research Center (UNRC), Department of Urology, Shahid Labbafinejad Medical Center, Shahid Beheshti University of Medical Sciences, Boostan $9^{\text {th }}$ Ave, Pasdaran St., Tehran, Iran; Phone: +98 21225672 22; Fax: +98 21225672 82;

e-mail:b_narouie@yahoo.com 
group versus rectus facia pubovaginal sling $(\mathrm{P}=0.007)$. Twenty eight $(80 \%)$ and $23(67 \%)$ patients in the mini sling (Ophira) and rectus facia pubovaginal sling were satisfied with the operation $(P=0.23)$. There is no significant difference between the mini sling (Ophira) and autologous rectus fascia sling procedure in the treatment of stress urinary incontinence at medium-term follow-up.

\section{Introduction}

Stress urinary incontinence (SUI) is the most common complaint of adult women with reported prevalence of up to $40 \%$ in large epidemiological surveys (Mostafa et al., 2012). SUI has a negative impact on quality of life and represents an important economic burden, as a social health problem (Gauruder-Burmester and Popken, 2009). Conservative managements such as pelvic floor muscle training, biofeedback and electrical stimulation may improve the problem, though persistence in therapy is needed (Sharifiaghdas and Mortazavi, 2008). However, for most of the women, surgery remains the basis of SUI management (Zoorob and Karram, 2012). Over the past decade, rectus facia pubovaginal sling has been introduced as the gold standard approach with high success rate even in the complicated and recurrent cases. However, the complications associated with this approach (e.g. bladder perforation, bleeding and wound infection) are still noted in up to $20 \%$ of the cases (Blaivas and Chaikin, 2011).Various minimally invasive procedures such as mid-urethral mini slings have been used recently in the treatment of SUI to overcome the possible morbidities. In these procedures, passage of the trocars through the retropubic or trans-obturator space is avoided with the intent to decrease postoperative pain and potential adverse events (Taub et al., 2005). Ophira mini sling system (Promedon, Argentina) is a rather new device which places a mid-urethral tension free polypropylene tape anchored to the urethropelvic ligament (retropubic approach) or obturator internus muscles bilaterally at the level of tendinous arc. The anchoring system is a specially designed fish bone like system at the end of each arm.

It is told that this system offers a very low perioperative complication rate, reduced hospital stay and operative time without jeopardizing the success rate (Abdel-Fattah et al., 2012). A few studies evaluated the efficacy and safety of this procedure in the treatment of SUI (Abdel-Fattah et al., 2011), however there is no randomized clinical trial report on this procedure in comparison with other gold standard techniques.

In this clinical trial, the safety and efficacy of Ophira mini sling was compared to pubovaginal rectus facia sling as a gold standard surgery in the treatment of women suffering from SUI.

\section{Material and Methods}

From January 2009 to December 2011, seventy two patients with the main complaint of SUI were enrolled in a prospective study. The inclusion criteria 
was: history of SUI, unresponsive to conservative managements, with urethral hypermobility and a positive cough stress test at equal to or more than $300 \mathrm{ml}$ bladder capacity. Preoperative evaluation consisted of: physical examination including pelvic floor evaluation in the lithotomy position, cough stress test, Q-tip test.

Multi-channel urodynamic study and cystourethroscopy was performed in all. Paraclinic imaging and tests including urinary tract ultrasound scan, complete blood count $(C B C)$, renal and liver function tests, serum electrolytes, urine analysis and culture was done in all.

Symptoms were scored according to the Incontinence Impact Questionnaire (IIQ) of Shumaker and colleagues (1994).

The degree of pelvic floor weakness was scored according to the pelvic organ prolapse quantification system (POP-Q) (Palma et al., 2010, 2012, 2013).

Hypermobility was defined as more than or equal to 45 degree change in the angle of urethra to horizontal line. Baseline demographic data including age, parity, menopausal status, body mass index (BMI), past medical history and pelvic surgeries were recorded.

Exclusion criteria were: persistent urinary tract infection (UTI) or active UTI at the time of surgery, evidences of urogynecological malignancies, cystocele grade of equal to or more than 3 , history of neurogenic bladder, abnormal filling or voiding phase in the urodynamic study (low capacity, low compliance, or detrusor overactivity, maximum flow rates of lower than $15 \mathrm{ml} / \mathrm{s}$ and residual urine of more than 100 milliliters), abnormal cystourethroscopy findings (trabeculated bladder, and or open bladder neck). The clinical study was approved by the Ethics Committee of the Urology and Nephrology Research Center (UNRC) of Shahid Labbafinejad Medical Center. Patients were randomly assigned to each group of Ophira mini sling versus standard rectus facia pubovaginal sling by envelope sealed cards.

\section{Surgical technique}

All procedures were carried out under spinal anesthesia in a lithotomy position. An experienced urologist ( $F$. Sharifiaghdas) accomplished all the operations in the pubovaginal sling group; after creation of Pfannenstiel skin incision; a 6-8 cm $\times 1-1.5 \mathrm{~cm}$ strip of the anterior rectus facia sheet was harvested. At the next steps, two parallel longitudinal incisions of about $1-1.5 \mathrm{~cm}$ were made on the anterior vaginal wall mucosa; at the level of the proximal urethra and lateral to the bladder neck.

After creation of a submucosal tunnel, the harvested facial graft was passed through the tunnel, and placed beneath the vaginal mucosa. The arms of graft were sutured by vicryl at the both ends and brought to the retropubic area. The long ends of vicryl sutures were tied to each other in the midline securing the sling in place without any tension ( 2 finger placed under the suprapubic midline knot). The anterior vaginal wall was checked to prevent overcorrection (the anterior vaginal 
wall should not move to the superior or posterior directions). At the end of the procedure, a 16-French Foley catheter was passed through the urethra and fixed in place. The urethral catheter was removed 1-3 days postoperative after the first attempts to void, residual urine was measured by clean catheterization and the patient was discharged if post-void residual urine was less than $100 \mathrm{ml}$.

In the Ophira mini sling group, a vertical incision of $1 \mathrm{~cm}$ was made on the anterior vaginal wall mucosa at the level of the mid urethra.

A gentle minimal dissection was made towards the inferior ramus of pubic bone. The delivery trocar was attached to the arms of mini sling mesh, guided by the surgeon's index finger towards the urethra-pelvic ligament. When half the length of the mesh was within the incision, the deploying button at the handle of the trocar was retracted to make a strong fixation of the mini sling mesh in the place. The same steps were repeated on the other side.

Foley catheter was left in place and removed 6 to 48 hours postoperative. All patients in both groups underwent cystourethroscopy during the procedure to rule out urethral or bladder penetration.

The patients were visited and followed-up regularly at one week and 1, 3,6 and 12 months postoperative.

At each visit they were examined and re-evaluated by objective measures (cough stress test with a full bladder) and subjective means (IIQ scores). The women were asked to describe their satisfaction from the surgery, on a visual analogue scale of $0-10$, whether or not they would have the procedure repeated if their incontinence returned, and whether they would recommend the procedure to a friend. A visual analogue scale of 8-10 and positive answers to another two questions were defined as satisfaction. The complications were recorded. Urine culture and ultrasound was repeated at each visit. Residual urine of more than $100 \mathrm{ml}$ was considered significant. Data analysis was performed by using SPSS software (Statistical Package for the Social Sciences, v16.0; SPSS Inc., Chicago, IL, USA). Student's $t$-test and chi-square test were used. A P-value of $<0.05$ was considered as statistically significant.

\section{Results}

Seventy two patients with the main complaint of SUI were followed for a mean time of $13.8 \pm 4.4$ months (12-20 months range).

Demographic and clinical characteristics of patients are shown in Table 1. The average patient's age $( \pm S D)$ in the sling and Ophira group were $52.2 \pm 9.3$ and $55.6 \pm 9.8$ years, respectively $(P=0.13)$.

There was no statistically significant difference between two groups regarding parameters including: weight, duration of urinary incontinence, number of vaginal deliveries, past obstetric history, hysterectomy and incontinence surgery. In terms of the preoperative functional results mean scores $( \pm S D)$ of Valsalva leak point pressure $(\mathrm{VLPP})$ and maximum flow rate $\left(\mathrm{Q}_{\max }\right)$ were $97 \pm 33.8 \mathrm{~cm} \mathrm{H} \mathrm{H}_{2} \mathrm{O}$ vs. 
Table 1 - Demographic data of patients in two groups of patients treated by rectus fascia sling versus mini sling Ophira device

\begin{tabular}{lccc}
\hline Variable & $\begin{array}{c}\text { Ophira group } \\
(\mathrm{n}=35)\end{array}$ & $\begin{array}{c}\text { Sling group } \\
(\mathrm{n}=35)\end{array}$ & P-value \\
\hline Age average (year) $( \pm \mathrm{SD})$ & $55.6 \pm 9.8$ & $52.2 \pm 9.3$ & 0.13 \\
Weight average $(\mathrm{kg})( \pm \mathrm{SD})$ & $71.7 \pm 11.4$ & $72.4 \pm 9.8$ & 0.77 \\
C/S history $(\%)$ & $9(25.7 \%)$ & $9(24.3 \%)$ & 0.65 \\
NVD (min-max) & $3(0-6)$ & $4(1-8)$ & 0.66 \\
Duration of incontinency & $2.4 \pm 1.5$ & $2.8 \pm 2.1$ & 0.37 \\
average (year) ( $\pm \mathrm{SD})$ & & & \\
Hysterectomy $(\%)$ & $3(8.6 \%)$ & $3(8.1 \%)$ & 0.10 \\
Incontinency surgery $(\%)$ & $1(2.9 \%)$ & $3(8.1 \%)$ & 0.61 \\
Marshal positive test $(\%)$ & $29(82.9 \%)$ & $34(91.1 \%)$ & 0.30 \\
VLPP average $( \pm S D)$ & $100.7 \pm 37.5$ & $97.0 \pm 33.8$ & 0.66 \\
$\mathrm{Q}_{\max }$ average (ml/s) $( \pm \mathrm{SD})$ & $27.2 \pm 6.5$ & $25.0 \pm 5.9$ & 0.14 \\
Average IIQ score (min-max) & $67.3(50-80)$ & $69.9(49-87)$ & 0.34 \\
\hline
\end{tabular}

$\mathrm{C} / \mathrm{S}$ - caesarean section; NVD - normal vaginal delivery;VLPP - Valsalva leak point pressure; $\mathrm{Q}_{\max }-$ maximum flow rate; IIQ - incontinence impact questionnaire

$100.7 \pm 37 \mathrm{~cm} \mathrm{H} \mathrm{H}_{2} \mathrm{O}(\mathrm{P}=0.66)$ and $25 \pm 5.9 \mathrm{ml} / \mathrm{s}$ vs. $27.2 \pm 6.5 \mathrm{ml} / \mathrm{s}(\mathrm{P}=0.14)$ in the sling and Ophira groups, respectively (Table 1).

Table 2 shows the detailed perioperative variables as well as the peri- and post-operative complications associated with both procedure the operation time was $65.6 \pm 22.6$ minutes vs. $12.9 \pm 11.3$ minutes in the sling and Ophira groups, respectively $(P=0.0001)$. Postoperative hospital stay was $36.6 \pm 14.4$ hours vs. $7.8 \pm 1.1$ hours, respectively $(P=0.0001)$.

The mean \pm SD Foley catheterization time in the sling and Ophira groups was $32.4 \pm 12.5$ and $10.8 \pm 2.3$ hours, respectively $(\mathrm{P}=0.0001)$.

Five $(13.5 \%)$ and six $(16.2 \%)$ patients in the sling group complained of urge incontinence and obstructive voiding symptoms respectively, while those complaints were recorded for one patient of Ophira group (2.9\%). All patients with obstructive symptoms were managed conservatively at first (anti-inflammatory drugs, $\mathrm{ClC}$ ). Urethral dilation was performed for 1 patient. No patient was under clean intermittent catheterization. Four patients in each group reported some degrees of SUl at one year post operation. Cough stress test was positive in $4(10.81 \%)$ and $4(11.42 \%)$ of the patients in the sling and Ophira groups respectively. There was no statistically significant difference between two groups in terms of dyspareunia, bladder perforation, urethral erosion, vaginal erosion-wound hematoma and/or infection was recorded in $21.6 \%$ and $2.9 \%$ of sling and Ophira group respectively $(\mathrm{P}=0.03)$, all treated conservatively (antibiotics, local care and dressings). Vaginal erosion of the mesh was observed in two cases (5.7\%) of Ophira group 7 and 8 months postoperative, treated by partial excision of the eroded tape and repair of vaginal wall. 
Table 2 - Peri- and post-operation patients' information in two groups of patients treated by rectus fascia sling versus mini sling Ophira device

\begin{tabular}{lccc}
\hline Variable & $\begin{array}{c}\text { Ophira group } \\
(\mathrm{n}=35)\end{array}$ & $\begin{array}{c}\text { Sling group } \\
(\mathrm{n}=35)\end{array}$ & P-value \\
\hline Time of surgery (min) $( \pm \mathrm{SD})$ & $12.9 \pm 11.3$ & $65.6 \pm 22.6$ & 0.0001 \\
Admit day average (hour) $( \pm \mathrm{SD})$ & $7.8 \pm 1.1$ & $59.9 \pm 11.4$ & 0.0001 \\
\hline & Complication & & \\
\hline Stress incontinence (\%) & $4(11.4 \%)$ & $4(10.8 \%)$ & 1.000 \\
Urge incontinence (\%) & $1(2.9 \%)$ & $5(13.5 \%)$ & 0.200 \\
Obstructive symptom (\%) & $1(2.9 \%)$ & $6(16.2 \%)$ & 0.100 \\
UTI (\%) & $0(0 \%)$ & $0(0 \%)$ & 1.000 \\
Hematoma and wound infection $(\%)$ & $1(2.9 \%)$ & $8(21.6 \%)$ & 0.030 \\
Pack cell infusion (\%) & $0(0 \%)$ & $1(2.7 \%)$ & 1.000 \\
Dyspareunia (\%) & $4(11.4 \%)$ & $3(8.1 \%)$ & 0.700 \\
Bladder perforation $(\%)$ & $0(0 \%)$ & $1(2.7 \%)$ & 1.000 \\
Urethral erosion $(\%)$ & $0(0 \%)$ & $0(0 \%)$ & 1.000 \\
Vaginal erosion (\%) & $2(5.7 \%)$ & $1(2.7 \%)$ & 0.610 \\
\hline & Average $/ 1 Q$ score $( \pm S D)$ & & \\
\hline Preoperative & $67.3 \pm 8.2$ & $69.6 \pm 9.6$ & 0.340 \\
Postoperative & $42.7 \pm 11.4$ & $50.2 \pm 11.1$ & 0.007 \\
P-value & 0.005 & 0.001 & \\
\hline
\end{tabular}

UTI - urinary tract infection; IIQ - incontinence impact questionnaire

Bladder penetration was discovered during the operation in one patient in the sling group which was managed by longer postoperative Foley catheterization (7 days).

Preoperative mean IIQ was 69.6 and 67.3 in the sling versus Ophira group which decreased to mean $50.2 \pm 11.1$ and $42.7 \pm 11.4$ after operation $(P=0.007)$. Twenty eight $(80 \%)$ and $25(67.5 \%)$ of the patients in the Ophira and sling group were satisfied with the operation $(\mathrm{P}=0.23)$.

\section{Discussion}

New generation of single incision mid-urethral slings were introduced from last decade with the intent to cure stress urinary incontinence in an outpatient with short operation time, less pain and less bleeding (Palma et al., 2010, 2012, 2013; Pushkar et al., 2010; Enzelsberger et al., 2011; Smith et al., 2011). Several clinical studies are ongoing to investigate the safety and efficacy of these new minimally invasive procedures compared to the gold standard surgeries, the Burch colposuspension and the pubovaginal slings with established high cure rates in the treatment of SUI. Despite high success rates with rectus facia pubovaginal sling even in complicated cases of SUI, there are some complications reported with this approach, such as wound infections, de novo urge incontinence and obstructive symptoms (Shumaker et al., 1994; Bump et al., 1996; Abrams et al., 2002; 
Athanasopoulos et al., 2011; Enzelsberger et al., 2011; Palma et al., 2012; Sivaslioglu et al., 2012). Ophira mini sling is among the new innovative systems, proposed to overcome these drawbacks, omit graft harvest and reduce excessive tension on bladder neck (Cody et al., 2003).

There are a few published case series on Ophira mini sling in the treatment of SUI. Palma et al. (2010) reported their experience on 124 female patients who suffered from SUI with a mean age $( \pm S D)$ of $54.7 \pm 9.9$ years old with a postoperative follow-up of at least 12 months. They used PAD test to objectively evaluate the surgical result. $81(85.3 \%)$ patients were cured, $6(6.3 \%)$ improved and 8 (8.4\%) remained incontinent (Palma et al., 2010). Pushkar and his colleagues (2010) published their results with Ophira in thirty women and reported a negative cough stress test in $92.8 \%$ at 12 months postoperative follow-up. Djehdian et al. (2010) compared the efficacy and safety of Ophira and trans-obturator mid-urethral tape (TOT) in a randomized trial, 45 and 28 women were followed. The author did not find any differences in subjective cure rates between two groups, however objectively the percentage of patients with postoperative negative cough stress test was statistically lower in Ophira group (Djehdian et al., 2010). Also, in a prospective study by Smith et al. (2011), patient satisfaction and clinical efficacy of two procedures Ophira and trans-obturator tape were compared. The authors concluded that Ophira and TOT procedures were comparable in terms of both objective and subjective cure rates; however the major advantage of Ophira compared to TOT was the facility of performing the procedure under local anesthesia on an ambulatory basis with less postoperative pain and intraoperative complication (Smith et al., 2011). Sharifiaghdas and Mortazavi (2008) have compared tension-free vaginal tape (TVT) with rectus facia pubovaginal sling in 61 patients ( 25 in the TVT and 36 in the sling group). The authors concluded that TVT and pubovaginal rectus facia sling were comparable in terms of both objective and subjective cure rate (Sharifiaghdas and Mortazavi, 2008). Our study showed an objective cure rate of $84.2 \%$ and $88.6 \%$ in the sling and Ophira groups respectively. Palma et al. (2012) proposed that the high load tolerated by Ophira mini sling is related to its multipoint fishbone-like polypropylene fixation device.

\section{Conclusion}

According to our data, it seems that the mini sling Ophira system and rectus facia pubovaginal sling procedures were comparable in terms of both objective and subjective cure rates. However the major advantages of Ophira compared to sling are shorter hospital stay, short duration of operation, less wound infection and more patient's satisfaction.

Acknowledgements:The authors thank to Urology and Nephrology Research Center (UNRC), Department of Urology, Shahid Labbafinejad Medical Center, Shahid Beheshti University of Medical Sciences, Tehran, Iran, for their helps in preparing this manuscript. 


\section{References}

Abdel-Fattah, M., Ford, J.A., Lim, C. P., Madhuvrata, P. (2011) Single-incision mini-slings versus standard midurethral slings in surgical management of female stress urinary incontinence: a meta-analysis of effectiveness and complications. Eur. Urol. 60, 468-480.

Abdel-Fattah, M., Agur, W., Abdel-All, M., Guerrero, K., Allam, M., Mackintosh, A., Mostafa, A., Yousef, M. (2012) Prospective multi-centre study of adjustable single-incision mini-sling $\left(A j u s t^{R}\right)$ in the management of stress urinary incontinence in women: 1-year follow-up study. BJU Int. 109(6), 880-886.

Abrams, P., Cardozo, L., Fall, M., Griffiths, D., Rosier, P., Ulmsten, U., van Kerrebroeck, P., Victor, A., Wein, A. (2002) The standardization of terminology of lower urinary tract function: report from Standardization Sub-committee of the International Continence Society. Neurourol. Urodyn. 21, 167-178.

Athanasopoulos, A., Gyftopoulos, K., McGuire, E. J. (2011) Efficacy and preoperative prognostic factors of autologous fascia rectus sling for treatment of female stress urinary incontinence. Urology 78(5), 1034-1038.

Blaivas, J. G., Chaikin, D. C. (2011) Pubovaginal fascial sling for the treatment of all types of stress urinary incontinence: surgical technique and long-term outcome. Urol. Clin. North Am. 38(1), 7-15.

Bump, R. C., Mattiasson, A., Bø, K., Brubaker, L. P., DeLancey, J. O., Klarskov, P., Shull, B. L., Smith, A. R. (1996) The standardization of terminology of female pelvic organ prolapse and pelvic floor dysfunction. Am. J. Obstet. Gynecol. 175, 10-17.

Cody, J., Wyness, L., Wallace, S., Glazener, C., Kilonzo, M., Stearns, S., McCormack, K., Vale, L., Grant, A. (2003) Systematic review of the clinical effectiveness and cost-effectiveness of tension-free vaginal tape for treatment of urinary stress incontinence. Health Technol. Assess. 7(21), 1-189.

Djehdian, L., Araujo, M., Takano, C., Del Roy, C., Castro, R., Sartori, M. G. F., Girão, M. J. B. C. (2010) Randomized trial of Ophira Mini-sling system and Unitape for the treatment of stress incontinence in women. First experiences after a follow up of 6 months. In: ICS/IUGA Meeting, Toronto, Canada, 2010.

Enzelsberger, H., Cemer, l., Kostersitz, E., Enzelsberger, S. (2011) Ophira-(Minisling) versus Monarc-(TOT) A prospective randomized study for the treatment of female stress urinary incontinence at a followup of 20 months. In: $36^{\text {th }}$ Annual Meeting of the International Urogynecological Association, Lisbon, Portugal, 2011.

Gauruder-Burmester, A., Popken, G. (2009) The MiniArc sling system in the treatment of female stress urinary incontinence. Int. Braz.J. Urol. 35(3), 334-341.

Mostafa, A., Agur, W., Abdel-All, M., Guerrero, K., Lim, C., Allam, M., Yousef, M., N'Dow, J., Abdel-Fattah, M. (2012) A multicentre prospective randomized study of single-incision mini-sling (Ajust ${ }^{R}$ ) versus tension-free vaginal tape-obturator (TVT-O ${ }^{\mathrm{TM}}$ ) in the management of female stress urinary incontinence: pain profile and short-term outcomes. Eur. J. Obstet. Gynecol. Reprod. Biol. 165(1), 115-121.

Palma, P., Riccetto, C., Bronzatto, E., Dalphorno, F., Herrmann, V., Altuna, S., Castro, R. (2010) Efficacy of Ophira mini sling system for stress urinary incontinence: Midterm follow up of patients in a multi-center international clinical trial. In: ICS/IUGA Meeting, Toronto, Canada, 2010.

Palma, P., Siniscalchi, R. T., Maciel, L. C., Bigozzi, M. A., Dal Fabbro, I., Riccetto, C. (2012) Primary fixation of mini slings: a comparative biomechanical study in vivo. Int. Braz. J. Urol. 38(2), 258-265; discussion 265-266.

Palma, P., Riccetto, C., Dalphorno, F., Castro, R., Altuna, S. (2013) Efficacy of Ophira mini sling system for stress urinary incontinence: Mid-term follow up of 176 patients in a multicentre international clinical trial. In: $41^{\text {st }}$ Annual ICS Meeting, Glasgow, UK, 2013.

Pushkar, D., Kasyan, G., Godunov, B., Gvozdev, M., Lynova, Y. (2010) Preliminary results of treatment of stress urinary incontinence with Ophira Mini-sling. In: ICS/IUGA Meeting, Toronto, Canada, 2010. 
Sharifiaghdas, F., Mortazavi, N. (2008) Tension-free vaginal tape and autologous rectus fascia pubovaginal sling for the treatment of urinary stress incontinence: a medium-term follow-up. Med. Princ. Pract. 17(3), 209-214.

Shumaker, S. A., Wyman, J. F., Uebersax, J. S., McClish, D., Fantl, J. A. (1994) Health-related quality of life measures for women with urinary incontinence:The Incontinence Impact Questionnaire and the Urogenital Distress Inventory. Continence Program in Women (CPW) Research Group. Qual. Life Res. 3, 291-306.

Sivaslioglu, A. A., Unlubilgin, E., Aydogmus, S., Keskin, L., Dolen, I. (2012) A prospective randomized controlled trial of the transobturator tape and tissue fixation mini-sling in patients with stress urinary incontinence: 5-year results. J. Urol. 188(1), 194-199.

Smith, P., Arunkalaivanan, A., Baptiste, M. (2011) Single-incision midurethral tape (Ophira ${ }^{\mathrm{TM}}$ ) vs. transobturator tape (Obtryx ${ }^{T M}$ ): Prospective comparative study at a median follow-up of 6 months. In: $36^{\text {th }}$ Annual Meeting of the International Urogynecological Association, Lisbon, Portugal, 2011.

Taub, D. A., Hollenbeck, B. K., Wie, J.T., Dunn, R. L., McGuire, E. J., Latini, J. M. (2005) Complications following surgical intervention for stress urinary incontinence: a national perspective. Neurourol. Urodyn. 24(7), 659-665.

Zoorob, D., Karram, M. (2012) Role of autologous bladder-neck slings: a urogynecology perspective. Urol. Clin. North Am. 39(3), 311-316. 